\title{
Collective Pairing of Resonantly Coupled Microcavity Polaritons
}

\author{
F. M. Marchetti ${ }^{1, *}$ and Jonathan Keeling ${ }^{2, \dagger}$ \\ ${ }^{1}$ Departamento de Física Teórica de la Materia Condensada and Condensed Matter Physics Center (IFIMAC), \\ Universidad Autónoma de Madrid, Madrid 28049, Spain \\ ${ }^{2}$ SUPA, School of Physics and Astronomy, University of St. Andrews, St. Andrews KY16 9SS, United Kingdom
}

(Received 5 August 2013; revised manuscript received 29 August 2014; published 21 November 2014)

\begin{abstract}
We consider the possible phases of microcavity polaritons tuned near a bipolariton Feshbach resonance. We show that, as well as the regular polariton superfluid phase, a "molecular" superfluid exists, with (quasi-)long-range order only for pairs of polaritons. We describe the experimental signatures of this state. Using variational approaches we find the phase diagram (critical temperature, density, and exciton-photon detuning). Unlike for ultracold atoms, the molecular superfluid is not inherently unstable, and our phase diagram suggests it is attainable in current experiments.
\end{abstract}

DOI: 10.1103/PhysRevLett.113.216405

PACS numbers: 71.36.+c, 03.75.Mn, 67.85.Fg

The wealth of physics explored with ultracold atomic gases [1] relies on the ability to tune parameters such as the interaction strength. A crucial tool to achieve this is the Feshbach resonance mechanism [2]: By using a magnetic field one may vary the detuning $\nu$ between two channels (hyperfine states) of the atoms, a closed channel (bound molecule) and an open (scattering) channel. When $\nu$ is large and positive, the closed channel is far above the open channel atoms, the formation of molecules is energetically suppressed, and atoms scatter with a weakly attractive effective interaction. When $\nu$ is large and negative, atoms are paired into molecules and the effective interaction is weakly repulsive. Near resonance $(\nu \simeq 0)$, the interaction is very large. This enables tunable pairing and regimes of strong correlations, allowing many interesting possibilities at both the few- and the many-body level.

Ultracold atom experiments are, however, intrinsically metastable, not true minima of the free energy. Three-body collisions must be avoided to prevent relaxation to, e.g., a solid phase. Also, Feshbach molecular states are highly rovibrationally excited states and can relax to lower states. Exploring regimes of strong interactions while suppressing such relaxation processes is inherently challenging. For fermionic atoms, Pauli exclusion suppresses scattering rates, so strongly interacting regimes can be accessed. In this case there is a smooth crossover between a BCS condensate of weakly attractive fermions and a BoseEinstein condensate (BEC) of repulsive molecules $[3,4]$.

The situation for bosons near a Feshbach resonance differs substantially: Unlike fermions, both a condensate of molecules and a condensate of unpaired bosons can exist. As discussed below, this means that a "molecular" superfluid phase with no off-diagonal long-range order (ODLRO) for atoms can arise, with a further symmetry breaking phase transition between "atomic" and "molecular" superfluids. Bosonic mixtures have attracted considerable theoretical interest [5-21]. However, experiments have been limited by stability issues: The molecular superfluid phase generally requires high densities $[13,15]$, where three-body losses are significant [22]. There have been suggestions to use optical lattices [8] to reach the strongly interacting regime while avoiding high densities, but to date, molecular pairing phases of bosonic atoms remain elusive.

Microcavity polaritons $[23,24]$, the quasiparticles resulting from strong coupling between cavity photons $(C)$ and quantum well excitons $(X)$, do not suffer the metastability problems of cold atoms and so present a more promising venue to study bosonic pairing phases. Microcavity polaritons have been observed to form a BEC $[25,26]$, and, as recently discussed $[27,28]$, interactions between polaritons with opposite polarizations can support either a bound state (bipolariton) or a scattering resonance depending on the exciton-photon detuning. Several signatures of this physics have been seen [29-31], including recent direct observations of the scattering resonance versus detuning [32,33]. Polaritons are expected not to suffer from the issues of three-body inelastic losses $[13,15,22]$ which plague cold bosonic atoms: Unlike ultracold atoms, a polariton BEC is not a metastable state, rather it is the minimum free-energy state as long as the polariton population is conserved. In addition, there is no deeply bound molecular state below the bipolariton. Polaritons do have a finite lifetime; however, recent experiments have demonstrated a five- to tenfold increase in lifetime [34], leading to a system very close to thermal equilibrium. A full nonequilibrium treatment of spin dynamics and relaxation is beyond the scope of this work. However, unlike cold atoms, such losses are not intrinsically linked to the resonance physics, and so they can be addressed and improved independently. Thus, polaritons may form a more promising venue to study pairing phases.

In this Letter we explore the phase diagram of collective paired phases arising from a bipolariton resonance in microcavities. By using the cavity-exciton detuning to tune the interactions $[27,28]$, we reveal a phase transition between atomic (i.e., polariton) and molecular (i.e., bipolariton) BEC phases. We show that temperatures and detunings required 
for typical materials such as GaAs are attainable and we discuss experimental signatures for detecting such phases.

Model.-In the model we consider, bipolaritons play the role of closed channel molecules, while the two (polarized) lower polariton (LP) modes are the open channel modes. The interchannel detuning is $\nu=2\left(\omega_{0}^{X}-\omega_{0}^{\mathrm{LP}}\right)-\left|E_{b}\right|$ (see Fig. 1), with $E_{b}$ the biexciton binding energy and $2 \omega_{\mathbf{k}}^{\mathrm{LP}}=$ $\omega_{\mathbf{k}}^{C}+\omega_{\mathbf{k}}^{X}-\sqrt{\left(\omega_{\mathbf{k}}^{C}-\omega_{\mathbf{k}}^{X}\right)^{2}+\Omega_{R}^{2}}$, where both the exciton and cavity photon dispersions are quadratic, $\omega_{\mathbf{k}}^{C, X}=$ $\omega_{0}^{C, X}+\left(k^{2} / 2 m_{C, X}\right)$. In fact, as discussed later and in Ref. [35], the bipolariton bound state is almost identical to a biexciton. However, it possesses a nonzero photon component, and for this reason we refer to it as a bipolariton. Even a small photon content has important consequences in breaking the degeneracy between dark and bright excitonic states [35]. The interchannel detuning $\nu$ can be controlled by varying the cavity-exciton detuning $\delta=\omega_{0}^{C}-\omega_{0}^{X}$ (see Fig. 1). A resonant enhancement of the interactions occurs near the bare resonance $\nu \simeq 0$.

We derive the many-body properties of resonantly coupled polaritons by considering a two-channel model, which includes both LPs in the right- and left-circular polarization basis, $\hat{\psi}_{\uparrow, \downarrow}$, and bipolariton $\hat{\psi}_{m}$ fields. One could, alternatively, work with a single-channel model with no explicit bipolariton field, at the expense of needing a finite range attractive potential $U^{\uparrow \downarrow}(\mathbf{r})$ supporting a resonant bound state [40]. Such an approach unnecessarily complicates the derivation of many-body physics, while a single-channel model with a contact potential cannot describe deeply bound bipolariton states.

The effective two-channel polariton model has been derived starting from a model describing coupled exciton,

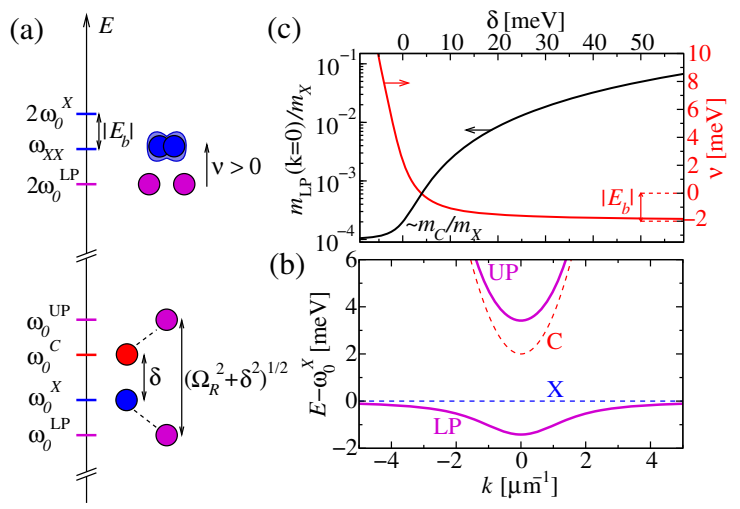

FIG. 1 (color online). (a) Illustration of one- and two-particle energy scales, showing how the cavity-exciton detuning $\delta$ determines the interchannel detuning $\nu$. (b) Lower (LP) and upper (UP) polariton dispersions [solid (magenta) lines], along with the cavity $C$ (exciton $X$ ) dispersion [dashed [red (blue)] lines] for $\delta=2 \mathrm{meV}$. (c) Dependence of the rescaled polariton mass at zero momentum $m_{\mathrm{LP}}(\mathbf{k}=0) / m_{X}$ [left (black) line] and of the interchannel detuning $\nu$ [right (red) line] on $\delta$. Plots are for GaAs parameters $\left(\Omega_{R}=4.4 \mathrm{meV},\left|E_{b}\right|=2 \mathrm{meV}\right.$ [27], photon mass $m_{C}=10^{-4} m_{X}$, exciton mass $m_{X}=0.4 m_{e}$ ). biexciton, and photon fields and rotating to the LP $\hat{\psi} \uparrow, \downarrow$ and bipolariton $\hat{\psi}_{m}$ fields [see the scheme in Fig. 1(a)]. This yields the following grand-canonical Hamiltonian written in momentum space, $\hat{\psi}_{\sigma}(\mathbf{r})=\sum_{\mathbf{k}} e^{i \mathbf{k} \cdot \mathbf{r}} \hat{a}_{\mathbf{k} \sigma} / \sqrt{\mathcal{A}}$ [where $\mathbf{k}$ is the in-plane (2D) momentum, $\mathcal{A}$ the system area, and $\hbar=1$ throughout]:

$$
\begin{aligned}
\hat{H} & =\sum_{\sigma=\uparrow, \downarrow, m} \sum_{\mathbf{k}} \hat{a}_{\mathbf{k} \sigma}^{\dagger}\left(\epsilon_{\mathbf{k} \sigma}-\mu_{\sigma}\right) \hat{a}_{\mathbf{k} \sigma} \\
& +\frac{1}{\mathcal{A}} \sum_{\sigma \sigma^{\prime}} \sum_{\mathbf{k}, \mathbf{k}^{\prime}, \mathbf{q}} \frac{U_{\mathbf{k}^{\prime} \mathbf{k}^{\prime} \mathbf{q}}^{\prime \mathbf{q}^{\prime}}}{2} \hat{a}_{\mathbf{k} \sigma}^{\dagger} \hat{a}_{\mathbf{k}^{\prime} \sigma^{\prime}}^{\dagger} \hat{a}_{\mathbf{k}^{\prime}-(\mathbf{q} / 2) \sigma^{\prime}} \hat{a}_{\mathbf{k}+(\mathbf{q} / 2) \sigma} \\
& +\frac{1}{\sqrt{\mathcal{A}}} \sum_{\mathbf{k}, \mathbf{Q}} \frac{g_{\mathbf{k} \mathbf{Q}}}{2}\left(\hat{a}_{\mathbf{k}+(\mathbf{Q} / 2) \uparrow}^{\dagger} \hat{a}_{-\mathbf{k}+(\mathbf{Q} / 2) \downarrow}^{\dagger} \hat{a}_{\mathbf{Q} m}+\text { H.c. }\right) .
\end{aligned}
$$

Here $\epsilon_{\mathbf{k} \uparrow, \downarrow}=\omega_{\mathbf{k}}^{\mathrm{LP}}-\omega_{0}^{\mathrm{LP}}$ is the full LP dispersion [see Fig. 1(b)]. The interchannel coupling coupling $g_{\mathbf{k} \mathbf{Q}}$ and the closed channel (bipolariton) dispersion can be derived by including the exciton-photon coupling in the exciton $T$ matrix, following Ref. [27] (see Ref. [35] for details). Because $m_{C} \ll m_{X}$, coupling to photons only weakly renormalizes the scattering resonance properties; hence, the bipolariton and biexciton dispersions almost coincide, so that $\epsilon_{\mathbf{k} m}=\left(k^{2} / 2 m_{m}\right)$ with $m_{m} \simeq 2 m_{X}$. In the absence of a magnetic field, the $\uparrow$ and $\downarrow$ populations are equal and the effective chemical potentials are $\mu_{\uparrow}=\mu_{\downarrow}=\mu$ and $\mu_{m}=2 \mu-\nu$, with $\nu$ as discussed above. The case with $\mu_{\uparrow} \neq \mu_{\downarrow}$ will be the subject of future study. Another question for future work is how the grand-canonical picture is modified in an open-dissipative framework.

To account for the varying excitonic fraction along the LP dispersion, the interaction terms include the Hopfield coefficient, $2 c_{\mathbf{k}}^{2}=1+\left(\omega_{\mathbf{k}}^{C}-\omega_{\mathbf{k}}^{X}\right) / \sqrt{\left(\omega_{\mathbf{k}}^{C}-\omega_{\mathbf{k}}^{X}\right)^{2}+\Omega_{R}^{2}}$. One finds that $g_{\mathbf{k} \mathbf{Q}}=c_{\mathbf{k}+(\mathbf{Q} / 2)} c_{-\mathbf{k}+(\mathbf{Q} / 2)} \sqrt{\Delta^{X} / m_{X}}$, where $\Delta^{X} \simeq$ $4\left|E_{b}\right|$ [27] is the excitonic resonance width [40]. The equivalent polaritonic energy scale $\Delta_{\mathbf{k Q}}^{\mathrm{LP}}=m g_{\mathbf{k} \mathbf{Q}}^{2}$ is given in terms of the LP mass at zero momentum $m=m_{\mathrm{LP}}(\mathbf{k}=$ $0)=\left[c_{0}^{2} / m_{X}+\left(1-c_{0}^{2}\right) / m_{C}\right]^{-1}$ [see Fig. 1(c)]. Similarly, the polariton interaction strengths also depend on the Hopfield coefficients [41], $U_{\mathbf{k k}^{\prime} \mathbf{q}}^{\sigma \sigma^{\prime}}=\tilde{U}_{\mathbf{k k}^{\prime} \mathbf{q}}^{\sigma \sigma^{\prime}} / m=$ $c_{\mathbf{k}} c_{\mathbf{k}^{\prime}} c_{\mathbf{k}^{\prime}-(\mathbf{q} / 2)} c_{\mathbf{k}+(\mathbf{q} / 2)} \tilde{U}_{X}^{\sigma \sigma^{\prime}} / m_{X}$, where $\tilde{U}^{\sigma \sigma^{\prime}}$ and $\tilde{U}_{X}^{\sigma \sigma^{\prime}}$ are dimensionless constants. Note that the parameters $U^{\uparrow \uparrow}=U^{\downarrow \downarrow}, 2 U^{\uparrow \downarrow}$, and $U^{m m}$ are the background (i.e., far from resonance) interaction strengths. Near resonance, the physical interaction also includes effects of the hybridization $g_{\mathbf{k} \mathbf{Q}}$. To estimate experimentally relevant parameters, we consider GaAs microcavities (as in Fig. 1), for which $\Omega_{R}=4.4 \mathrm{meV}, \quad\left|E_{b}\right|=2 \mathrm{meV}$ [27], $m_{C}=10^{-4} m_{X}$, $m_{X}=0.4 m_{e}$, the resonance $\nu=0$ is at $\delta=3.84 \mathrm{meV}$, and $\tilde{U}_{X}^{\uparrow \uparrow}=\tilde{U}_{X}^{\downarrow \downarrow}=6$, and we take $\tilde{U}^{\uparrow \downarrow}=0$ [27]. Because $m_{C} \ll m_{X}$, the LP fluid is much more weakly interacting than the excitonic fluid, i.e., $\tilde{U}^{\sigma \sigma^{\prime}} \ll \tilde{U}_{X}^{\sigma \sigma^{\prime}}$, except when $\delta \gg$ $\Omega_{R}$ and the LP becomes pure exciton. As widely discussed for atoms $[6,13,15]$, repulsive closed-channel interactions, $\tilde{U}_{X}^{m m}>0$, are required for stability. Biexciton interactions 
are indeed repulsive, as discussed in the context of biexciton condensation, see, e.g., Ref. [42], and we take $\tilde{U}_{X}^{m m}=4$.

Zero temperature.-Figure 2 shows the $T=0$ phase diagram for the GaAs parameters specified above. The axes are the detuning $\delta$ and either the chemical potential $\mu$ or the total density, $n=\sum_{\mathbf{k}}\left\langle\hat{a}_{\mathbf{k} \uparrow}^{\dagger} \hat{a}_{\mathbf{k} \uparrow}+\hat{a}_{\mathbf{k} \downarrow}^{\dagger} \hat{a}_{\mathbf{k} \downarrow}+2 \hat{a}_{\mathbf{k} m}^{\dagger} \hat{a}_{\mathbf{k} m}\right\rangle / \mathcal{A}$. Details of the calculation appear below. Varying $\delta$ changes both the interchannel detuning $\nu$ and the LP dispersion $\omega_{k}^{\mathrm{LP}}$. Three phases exist [43]: the normal phase $(\mathrm{N}), \psi_{\uparrow}=$ $\psi_{\downarrow}=\psi_{m}=0$ (where $\psi_{\sigma}=\left\langle\hat{\psi}_{\sigma}\right\rangle=\left\langle\hat{a}_{0 \sigma}\right\rangle / \sqrt{\mathcal{A}}$ ) and two condensed phases arising from the spontaneous symmetry breaking of the $U(1) \times U(1)$ global symmetry,

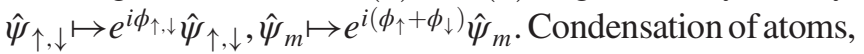
$\psi_{\uparrow}=\psi_{\downarrow} \neq 0$, guarantees condensation of molecules, $\psi_{m} \neq$ 0 (but not vice versa). We denote this phase as an atomic and molecular superfluid phase (AMSF): Here, the $U(1) \times U(1)$ symmetry is completely broken. The third phase is characterized by the absence of atomic ODLRO but where molecules do condense, the MSF phase [8-12]. The MSF phase has a residual $U(1)$ symmetry (rotations with $\phi_{\uparrow}+\phi_{\downarrow}=$ const).

For $\delta \lesssim 1.6 \mathrm{meV}$, Fig. 2 shows a second order N-AMSF transition, as expected when bipolaritons are irrelevant. As $\delta$ increases, $\nu$ decreases and the bare resonance occurs at $\delta=3.84 \mathrm{meV}$. The MSF phase appears at slightly higher $\delta \simeq 4.0 \mathrm{meV}$. This is because near the resonancebetween the lower two tricritical points [solid (blue) circles] - the N-AMSF phase transition becomes first order, leading to phase separation (right-hand panel of Fig. 2), a dramatic signature of the resonance effects. Phase separation occurs either between $\mathrm{N}$ and AMSF below the critical end point [solid (red) diamond at $\delta \simeq 4.0 \mathrm{meV}$ ] or above, between MSF and AMSF. Above the second tricritical point, there is a second order N-MSF transition at $2 \mu=\nu$, which is then followed by a second order MSF-AMSF transition. This MSF-AMSF transition occurs

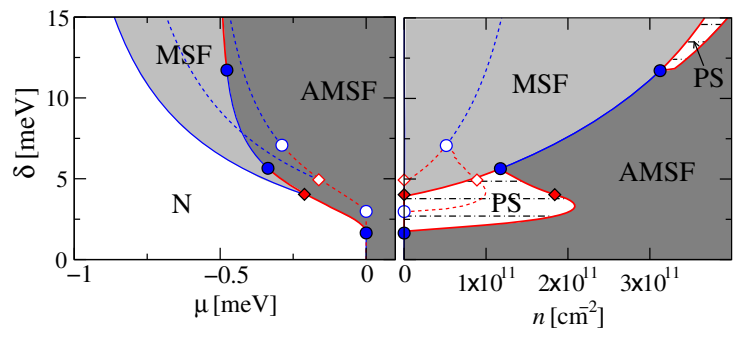

FIG. 2 (color online). $T=0$ phase diagram for a GaAs microcavity, plotting cavity-exciton detuning $\delta$ versus chemical potential $\mu$ (left) or total density $n$ (right). The normal phase $\mathrm{N}$ is white, MSF shaded light gray, and AMSF is dark gray. First [thick (red)] and second [thinner (blue)] order lines are separated by tricritical points (blue circles), while critical end points are (red) diamonds. Horizontal dot-dashed lines connect the same chemical potential first order boundaries in the phase separated (PS) region. Dashed lines are the mean-field boundaries neglecting quantum fluctuations. when the renormalized bipolariton energy (due to the interaction $\tilde{U}_{X}^{m m}$ ) reaches the polariton energy, as discussed in Ref. [13]. At even higher detunings, the MSF-AMSF transition becomes first order again, driven by the changing polariton dispersion (see Ref. [35]).

As well as clear signatures in the form of the phase diagram, the different collective paired phases can be experimentally identified via spatial correlation functions. In particular, at $T=0$, the AMSF phase is characterized by ODLRO of both unpaired polaritons and bipolaritons. At $T \neq 0$, as the system is $2 \mathrm{D}$, this evolves into off-diagonal quasi-long-range order, i.e., power-law decay of the correlation functions $g_{\uparrow, \downarrow}^{(1)}(\mathbf{r})=$ $\left\langle\hat{\psi}_{\uparrow, \downarrow}^{\dagger}(\mathbf{r}) \hat{\psi}_{\uparrow, \downarrow}(0)\right\rangle$ and $g_{m}^{(1)}(\mathbf{r})=\left\langle\hat{\psi}_{\uparrow}^{\dagger}(\mathbf{r}) \hat{\psi}_{\downarrow}^{\dagger}(\mathbf{r}) \hat{\psi}_{\downarrow}(0) \hat{\psi}_{\uparrow}(0)\right\rangle$. In contrast, the MSF phase is characterized by the absence of any order for unpaired polaritons, while displaying power-law decay of $g_{m}^{(1)}(\mathbf{r})$ : The observation of such pair correlations without polariton correlations would provide unambiguous evidence for a MSF phase. An experimental scheme to measure $g_{m}^{(1)}$ is given in Ref. [35].

To derive the $T=0$ phase diagram we employ a variational approach, by considering a normalized Bogoliubov-Nozières ground state [44] including atomic and molecular condensates, as well as pairing terms:

$|\psi\rangle=\mathcal{N} \exp \left(\sqrt{\mathcal{A}} \sum_{\sigma=\uparrow, \downarrow, m} \psi_{\sigma} \hat{a}_{0 \sigma}^{\dagger}+\sum_{\mathbf{k}} \sum_{\gamma=a, b, m} \tanh \theta_{\mathbf{k} \gamma} \hat{b}_{\mathbf{k} \gamma}^{\dagger} \hat{b}_{-\mathbf{k} \gamma}^{\dagger}\right)|0\rangle$.

The operators $\hat{b}_{\mathbf{k} \gamma}$ are related to $\hat{a}_{\mathbf{k} \sigma}$ by

$$
\left(\begin{array}{l}
\hat{a}_{\mathbf{k} \uparrow}^{\dagger} \\
\hat{a}_{\mathbf{k} \downarrow}^{\dagger}
\end{array}\right)=\frac{1}{\sqrt{2}}\left(\begin{array}{cc}
1 & 1 \\
-1 & 1
\end{array}\right)\left(\begin{array}{c}
\hat{b}_{\mathbf{k} a}^{\dagger} \\
\hat{b}_{\mathbf{k} b}^{\dagger}
\end{array}\right), \quad \hat{a}_{\mathbf{k} m}^{\dagger}=\hat{b}_{\mathbf{k} m}^{\dagger} .
$$

Note that $\sigma=\uparrow, \downarrow, m$, while $\gamma=a, b, m$. This transformation produces the most general ( $\uparrow \leftrightarrow \downarrow$ symmetric) variational ground state including pairing. Minimizing the energy $\langle\hat{H}\rangle$ over the variational parameters $\psi_{\uparrow}=$ $\psi_{\downarrow}=\psi_{0}, \psi_{m}$, and $\theta_{\mathbf{k} \gamma}$, we find that $\theta_{\mathbf{k} \gamma}$ has the functional form $\tanh 2 \theta_{\mathbf{k} \gamma}=\alpha_{\gamma} /\left(\epsilon_{\mathbf{k} \gamma}+\beta_{\gamma}\right)$, with $\beta_{\gamma}>0,\left|\alpha_{\gamma}\right| \leq \beta_{\gamma}$, and $\epsilon_{\mathbf{k} a, b}=\omega_{\mathbf{k}}^{\mathrm{LP}}-\omega_{0}^{\mathrm{LP}}$. The energy can thus be numerically minimized in terms of eight variational parameters, $\alpha_{\gamma}, \beta_{\gamma}$, and $\psi_{\sigma}$, making it easy to determine first order phase boundaries, as well as to find cases where the global minimum energy is not an extremum (zero derivative) but instead occurs at $\left|\alpha_{\gamma}\right|=\beta_{\gamma}$.

As $\tilde{U}^{\sigma \sigma^{\prime}} \ll \tilde{U}_{X}^{\sigma \sigma^{\prime}} \sim 1$, fluctuation corrections to mean-field (MF) theory should be small. At the same time, as bipolaritons have a much larger mass than LPs, bipolariton fluctuations give a non-negligible shift. The dashed lines and empty symbols in Fig. 2 show the MF predictions. Fluctuations do shift the phase boundaries, but the phase diagram topology qualitatively matches MF predictions [12], except for the tricritical point at large detuning where the MSF-AMSF transition becomes first order again (see Ref. [35]). At MF level, the two tricritical points are at 
$(\mu, \nu)=\left(0, \Delta_{00}^{\mathrm{LP}} /(4 \bar{U})\right)$ and $\left(-\Delta_{00}^{\mathrm{LP}} /(2 \bar{W}),-\Delta_{00}^{\mathrm{LP}}\left[\bar{W}^{-1}+\right.\right.$ $\left.\left.(8 \bar{U})^{-1}\right]\right)$, where $\bar{U}=\tilde{U}^{\uparrow \uparrow}+\tilde{U}^{\uparrow \downarrow}$ and $\bar{W}=\left[\tilde{U}^{m m}\left(\tilde{U}^{\uparrow \uparrow}+\right.\right.$ $\left.\left.\tilde{U}^{\uparrow \downarrow}\right)\right]^{1 / 2}$.

Finite temperature.-We have shown that, in GaAs, a polariton MSF phase can be found at $T=0$ for $\delta \gtrsim 4.0 \mathrm{meV}$. However, to see if such a phase is readily accessible, we must determine its critical temperature. Since the closed channel mass is $m_{M} \simeq 2 m_{X}$, the critical temperature is expected to be much lower than corresponding LP condensation temperatures.

In order to extend our results to finite temperature, we use variational mean-field theory (VMFT) [12,45], based on the inequality [46] $\mathcal{F}=-k_{B} T \ln \operatorname{Tr} e^{-\hat{H} / k_{B} T} \leq$ $\mathcal{F}_{v \mathrm{MF}}=\mathcal{F}_{\mathrm{MF}}+\left\langle\hat{H}-\hat{H}_{\mathrm{MF}}\right\rangle_{\mathrm{MF}}$. In a similar spirit to the $T=0$ calculation, $\hat{H}_{\mathrm{MF}}$ is chosen to allow the same oneand two-point correlation functions, and its variational parameters are used to minimize $\mathcal{F}_{v \mathrm{MF}}$ :

$$
\begin{aligned}
\hat{H}_{\mathrm{MF}}= & \sum_{\gamma=a, b, m}\left\{-\sqrt{\mathcal{A}} \psi_{\gamma}\left(\alpha_{\gamma}+\beta_{\gamma}\right)\left(\hat{b}_{0 \gamma}^{\dagger}+\hat{b}_{0 \gamma}\right)\right. \\
& \left.+\frac{1}{2} \sum_{\mathbf{k}}\left(\hat{b}_{\mathbf{k} \gamma}^{\dagger} \hat{b}_{-\mathbf{k} \gamma}\right)\left(\begin{array}{cc}
\epsilon_{\mathbf{k} \gamma}+\beta_{\gamma} & \alpha_{\gamma} \\
\alpha_{\gamma} & \epsilon_{\mathbf{k} \gamma}+\beta_{\gamma}
\end{array}\right)\left(\begin{array}{c}
\hat{b}_{\mathbf{k} \gamma} \\
\hat{b}_{-\mathbf{k} \gamma}^{\dagger}
\end{array}\right)\right\},
\end{aligned}
$$

where $\psi_{a}=0$ and $\psi_{b}=\sqrt{2} \psi_{0}$. As before, the functional form of the matrix elements above is the optimal form. We evaluate $\mathcal{F}_{\mathrm{MF}}$ and averages $\langle\cdot\rangle_{\mathrm{MF}}$ by standard Bogoliubov diagonalization [47]. This yields the free energy $\mathcal{F}_{v \mathrm{MF}}$ as a function of the same eight parameters $\alpha_{\gamma}, \beta_{\gamma}$, and $\psi_{0}, \psi_{m}$, again allowing numerical minimization. Note that the $T=0$ limit of this approach reproduces the results presented above.

For 2D Bose gases the actual $T \neq 0$ transition to a superfluid phase is of the Berezinskii-Kosterlitz-Thouless type [47]. Instead, VMFT predicts a first order transition. The N-AMSF and MSF-AMSF transitions can be very weakly first order due to the small polariton mass [35]. Despite the absence of true ODLRO, the quasicondensate density plays a similar role to the mean-field order parameter $[48,49]$. This allows Hartree-Fock [47,50] or equivalent approaches (such as VMFT) to reproduce the equation of state of the system outside of the critical region. As such, the location of phase boundaries predicted by VMFT should be accurate, and we have verified this for the homonuclear weakly interacting 2D Bose gas by comparison to the Monte Carlo results of Ref. [49]. In the 3D homonuclear case, other approaches, such as applications of the Nozières-Schmitt-Rink approximation [51], have been considered, predicting only second order transitions [15].

Figure 3 shows the phase diagram both versus $\delta$ at fixed $T$ (top panels) and versus $T$ at fixed $\delta$ (bottom panels). The main new features introduced by nonzero temperature are the existence of a nonzero critical density for the normal state and the extension to arbitrarily large detuning of

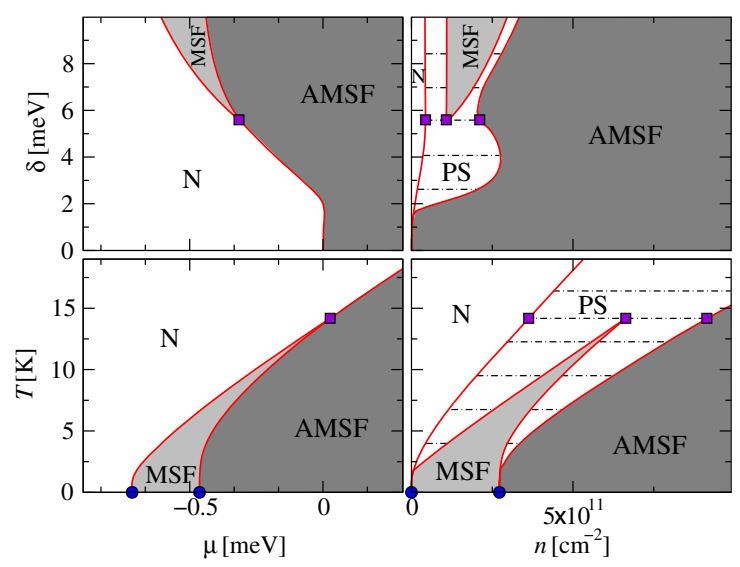

FIG. 3 (color online). Finite temperature phase diagrams with the same notation, color scheme, and GaAs parameters as in Fig. 2. The critical end point of Fig. 2 is now replaced by a triple point (purple square). Temperature is fixed to $T=4 \mathrm{~K}\left(k_{B} T=\right.$ $0.34 \mathrm{meV}$ ) in the top panels, while detuning is $\delta=10 \mathrm{meV}$ in the bottom ones.

the phase separated region as discussed above. Because the N-MSF transition is now first order, the critical end point at $T=0$ is replaced by a triple point. At yet higher temperatures, the triple point moves to higher $\delta$ and eventually merges with the remnant tricritical point [35]. In the phase diagram versus temperature at $\delta=10 \mathrm{meV}$, one sees that the MSF phase survives up to $T \sim 14.2 \mathrm{~K}(1.28 \mathrm{meV})$. For $\mathrm{ZnO}$ [52] the MSF phase can survive to higher temperatures and lower detunings [35]. In Ref. [35] we compare these results with those obtained for a quadratic approximation to the LP dispersion. The phase diagrams are qualitatively similar; however, the quadratic approximation incorrectly restricts the extent of the MSF phase.

Conclusions. - To conclude, we argue that microcavity polaritons are a particularly promising system to explore collective pairing phases of bosons. In particular, in addition to the standard polariton superfluid phase, where both polaritons and bipolaritons are characterized by offdiagonal quasi-long-range order, we highlight a new phase that displays molecular superfluidity, i.e., order for bipolaritons but not for polaritons. This phase covers an increasing region of the phase diagram at either larger cavity-exciton detunings or lower temperatures. While for the GaAs parameters considered here we predict the MSF phase to survive up to $T \sim 14 \mathrm{~K}$ at a cavity-exciton detuning $\delta=10 \mathrm{meV}$, this temperature can be higher for materials with larger Rabi splitting, such as $\mathrm{ZnO}$.

We are grateful to B.D. Simons, L. Radzihovsky, and M. Wouters for stimulating discussions. F. M. M. acknowledges financial support from the programs Ramón y Cajal, MINECO (MAT2011-22997), CAM (S-2009/ESP-1503), and Intelbiomat (ESF). J. K. acknowledges support from the EPSRC program "TOPNES" (EP/I031014/1) and EPSRC (EP/G004714/2). 
*francesca.marchetti@uam.es

†jmjk@st-andrews.ac.uk

[1] I. Bloch, J. Dalibard, and W. Zwerger, Rev. Mod. Phys. 80, 885 (2008).

[2] C. Chin, R. Grimm, P. Julienne, and E. Tiesinga, Rev. Mod. Phys. 82, 1225 (2010).

[3] W. Ketterle and M. W. Zwierlein, Nuovo Cimento Riv. Ser. 31, 247 (2008).

[4] A. J. Leggett and S. Zhang, The BEC-BCS Crossover: Some History and Some General Observations, edited by W. Zwerger, The BCS-BEC Crossover and the Unitary Fermi Gas, Vol. 836 (Springer, Berlin, 2012), pp. 33-47.

[5] E. Timmermans, P. Tommasini, M. Hussein, and A. Kerman, Phys. Rep. 315, 199 (1999).

[6] E. J. Mueller and G. Baym, Phys. Rev. A 62, 053605 (2000).

[7] G. S. Jeon, L. Yin, S. W. Rhee, and D. J. Thouless, Phys. Rev. A 66, 011603 (2002).

[8] A. Kuklov, N. Prokof'ev, and B. Svistunov, Phys. Rev. Lett. 92, 030403 (2004).

[9] A. Kuklov, N. Prokof'ev, and B. Svistunov, Phys. Rev. Lett. 92, 050402 (2004).

[10] L. Radzihovsky, J. Park, and P. B. Weichman, Phys. Rev. Lett. 92, 160402 (2004).

[11] M. W. J. Romans, R. A. Duine, S. Sachdev, and H. T. C. Stoof, Phys. Rev. Lett. 93, 020405 (2004).

[12] L. Radzihovsky, P. B. Weichman, and J. I. Park, Ann. Phys. (Amsterdam) 323, 2376 (2008).

[13] S. Basu and E. J. Mueller, Phys. Rev. A 78, 053603 (2008).

[14] L. Zhou, J. Qian, H. Pu, W. Zhang, and H. Y. Ling, Phys. Rev. A 78, 053612 (2008).

[15] A. Koetsier, P. Massignan, R. A. Duine, and H. T. C. Stoof, Phys. Rev. A 79, 063609 (2009).

[16] M. J. Bhaseen, M. Hohenadler, A. O. Silver, and B. D. Simons, Phys. Rev. Lett. 102, 135301 (2009).

[17] L. Radzihovsky and S. Choi, Phys. Rev. Lett. 103, 095302 (2009).

[18] M. Hohenadler, A. O. Silver, M. J. Bhaseen, and B. D. Simons, Phys. Rev. A 82, 013639 (2010).

[19] S. Ejima, M. J. Bhaseen, M. Hohenadler, F. H. L. Essler, H. Fehske, and B. D. Simons, Phys. Rev. Lett. 106, 015303 (2011).

[20] M. J. Bhaseen, S. Ejima, F. H. L. Essler, H. Fehske, M. Hohenadler, and B.D. Simons, Phys. Rev. A 85, 033636 (2012).

[21] F. Zhou and M. S. Mashayekhi, Ann. Phys. (Amsterdam) 328, 83 (2013).

[22] B. S. Rem, A. T. Grier, I. Ferrier-Barbut, U. Eismann, T. Langen, N. Navon, L. Khaykovich, F. Werner, D. S. Petrov, F. Chevy, and C. Salomon, Phys. Rev. Lett. 110, 163202 (2013).

[23] A. V. Kavokin, J. J. Baumberg, G. Malpuech, and F. P. Laussy, Microcavities (Oxford University Press, Oxford, England, 2007).

[24] I. Carusotto and C. Ciuti, Rev. Mod. Phys. 85, 299 (2013).

[25] J. Kasprzak, M. Richard, S. Kundermann, A. Baas, P. Jeambrun, J. M. J. Keeling, F. M. Marchetti, M. H. Szymaska, R. André, J. L. Staehli, V. Savona, P. B. Littlewood, B. Deveaud, and L. S. Dang, Nature (London) 443, 409 (2006).

[26] R. Balili, V. Hartwell, D. Snoke, L. Pfeiffer, and K. West, Science 316, 1007 (2007).

[27] M. Wouters, Phys. Rev. B 76, 045319 (2007).
[28] I. Carusotto, T. Volz, and A. Imamolu, Europhys. Lett. 90, 37001 (2010).

[29] M. Saba, F. Quochi, C. Ciuti, U. Oesterle, J. L. Staehli, B. Deveaud, G. Bongiovanni, and A. Mura, Phys. Rev. Lett. 85, 385 (2000).

[30] P. Borri, W. Langbein, and U. Woggon, Semicond. Sci. Technol. 18, S351 (2003).

[31] P. Wen, G. Christmann, J. J. Baumberg, and K. A. Nelson, New J. Phys. 15, 025005 (2013).

[32] N. Takemura, S. Trebaol, M. Wouters, M. T. Portella-Oberli, and B. Deveaud, Nat. Phys. 10, 500 (2014).

[33] N. Takemura, S. Trebaol, M. Wouters, M. T. Portella-Oberli, and B. Deveaud, arXiv:1310.6506.

[34] P. Wen, Y. Sun, K. A. Nelson, B. Nelsen, G. Liu, M. Steger, D. W. Snoke, L. N. Pfeiffer, and K. West, "Phase diagram of Bose condensation of long-lifetime polaritons in equilibrium" (to be published).

[35] See Supplemental Material at http://link.aps.org/ supplemental/10.1103/PhysRevLett.113.216405 for derivation of effective model, details of pair coherence detection scheme, and further cuts through phase diagram, as well as Refs. [36-39].

[36] A. Trichet, E. Durupt, F. Médard, S. Datta, A. Minguzzi, and M. Richard, Phys. Rev. B 88, 121407 (2013).

[37] H. W. van Kesteren, E. C. Cosman, W. A. J. A. van der Poel, and C. T. Foxon, Phys. Rev. B 41, 5283 (1990).

[38] M. Combescot, O. Betbeder-Matibet, and R. Combescot, Phys. Rev. Lett. 99, 176403 (2007).

[39] T. Makino, Y. Segawa, M. Kawasaki, and H. Koinuma, Semicond. Sci. Technol. 20, S78 (2005).

[40] V. Gurarie and L. Radzihovsky, Ann. Phys. (Amsterdam) 322, 2 (2007).

[41] M. Wouters and I. Carusotto, Phys. Rev. B 75, 075332 (2007).

[42] A. Mysryowicz, in Bose-Einstein Condensation, edited by A. Griffin, D. W. Snoke, and S. Stringari (Cambridge University Press, Cambridge, England, 1995), p. 330.

[43] For imbalanced populations in the presence of a Zeeman field, two additional phases are allowed [14]: $\psi_{\downarrow} \neq 0$, $\psi_{\downarrow}=0, \psi_{m}=0$ and $\psi_{\uparrow}=0, \psi_{\downarrow} \neq 0, \psi_{m}=0$.

[44] P. Noziéres and D. Saint James, J. Phys. (Paris) 43, 1133 (1982).

[45] H. Kleinert, Path Integrals in Quantum Mechanics, Statistics and Polymer Physics (World Scientific, Singapore, 1995).

[46] R. P. Feynman, Statistical Mechanics: A Set Of Lectures (Advanced Books Classics) (Westview Press, Boulder, 1998) p. 368.

[47] L. P. Pitaevskii and S. Stringari, Bose-Einstein Condensation (Clarendon Press, Oxford, England, 2003).

[48] Y. Kagan, V. A. Kashurnikov, A. V. Krasavin, N. V. Prokof'ev, and B. V. Svistunov, Phys. Rev. A 61, 043608 (2000).

[49] N. Prokof'ev and B. Svistunov, Phys. Rev. A 66, 043608 (2002).

[50] V. N. Popov, Functional Integrals in Quantum Field Theory and Statistical Physics (D. Reidel, Dordrecht, 1983).

[51] P. Nozieres and S. Schmitt-Rink, J. Low Temp. Phys. 59, 195 (1985).

[52] F. Li, L. Orosz, O. Kamoun, S. Bouchoule, C. Brimont, P. Disseix, T. Guillet, X. Lafosse, M. Leroux, J. Leymarie, M. Mexis, M. Mihailovic, G. Patriarche, F. Réveret, D. Solnyshkov, J. Zuniga-Perez, and G. Malpuech, Phys. Rev. Lett. 110, 196406 (2013). 